Meta

Journal des traducteurs

Translators' Journal

\title{
Pigistes et bureaux de traduction
}

\section{Roger Boivineau}

Volume 21, numéro 1, mars 1976

La traduction et l'entreprise

URI : https://id.erudit.org/iderudit/002099ar

DOI : https://doi.org/10.7202/002099ar

Aller au sommaire du numéro

Éditeur(s)

Les Presses de l'Université de Montréal

ISSN

0026-0452 (imprimé)

1492-1421 (numérique)

Découvrir la revue

Citer cet article

Boivineau, R. (1976). Pigistes et bureaux de traduction. Meta, 21(1), 5-11.

https://doi.org/10.7202/002099ar d'utilisation que vous pouvez consulter en ligne.

https://apropos.erudit.org/fr/usagers/politique-dutilisation/ 


\section{Pigistes et bureaux de traduction}

En abordant une étude, même très succincte, d'un secteur de l'activité économique, il est bon de le situer quantitativement. Certes, la traduction ne représente pas une part énorme du produit national brut, bien qu'elle y occupe sans aucun doute une place plus importante au Canada que dans la plupart des pays, mais il serait intéressant de connaître au moins le nombre de bureaux de traduction existant au Canada, leur chiffre d'affaires et le nombre de traducteurs qu'ils emploient. Malheureusement, la traduction, souvent largement méconnue, semble de plus être inconnue des statisticiens. Apparemment, il n'existe pas de statistiques fournissant les quelques données qui permettraient de mieux évaluer notre secteur. On en est donc réduit à des estimations « à vue de nez », qui n'ont certes rien de scientifique ni, par conséquent, de précis.

\section{LE MARCHÉ}

Le marché se répartit, selon la division classique, entre le secteur public et le secteur privé. Le secteur public est essentiellement composé des administrations. Le gouvernement fédéral, qui est, de très loin, le principal employeur de traducteurs, est aussi sans aucun doute le plus gros acheteur de traduction; ses services spécialisés ne suffisant pas à la tâche.

Les gouvernements provinciaux, du Québec, principalement pour la traduction du français à l'anglais, de l'Ontario et du Nouveau-Brunswick, sont également acheteurs de traduction. Il faut y ajouter les organismes paragouvernementaux et sociétés de la Couronne. Généralement ces acheteurs préfèrent traiter avec des pigistes, pour des raisons de tarif.

Le secteur privé se compose d'entreprises appartenant à peu près à tous les domaines de l'activité économique et aussi au secteur culturel.

Comme dans le cas du gouvernement, certaines grandes entreprises ont déjà leur service de traduction mais envoient à l'extérieur le surplus de travail. Elles s'adressent aussi bien aux pigistes qu'aux maisons de traduction. Le secteur de l'édition semble plutôt se tourner vers les pigistes spécialisés, pour la traduction des livres et articles. 
L'aire géographique du marché de la traduction est évidemment calquée sur la carte administrative et économique du pays.

Les grands marchés de la traduction se trouvent à Ottawa, centre administratif, Montréal et Toronto, centres économiques.

\section{LES FOURNISSEURS}

Faute de chiffres plus précis, l'examen des pages publicitaires de l'annuaire du téléphone permet de dénombrer environ 70 bureaux de traduction à Montréal et environ 50 à Toronto. Ces maisons se spécialisent pour la plupart dans la traduction de l'anglais et du français, mais certaines n'hésitent pas toutefois à annoncer «traduction dans toutes les langues». Quatre ou cinq bureaux sont établis à la fois à Montréal et à Toronto mais, dans la plupart des cas, un des deux établissements n'est en fait qu'un bureau de représentation.

Quant au nombre de traducteurs employés par ces maisons, il ne peut être évalué que par des approximations très vagues. Par exemple, certains bureaux n'occupent même pas un seul traducteur permanent et font plutôt office de courtiers en traduction, leur tâche se limitant à transmettre à leurs clients les traductions qu'ils ont fait exécuter par un de leurs pigistes. Plusieurs autres bureaux sont ceux d'un traducteur « solitaire » travaillant à son compte.

À l'autre extrémité de l'éventail, se trouvent les bureaux de traduction relativement grands, c'est-à-dire employant plus de cinq traducteurs en permanence. Il ne semble pas que ce nombre de traducteurs permanents soit dépassé de beaucoup... même si un bureau annonce qu'il met plus de 2000 spécialistes à la disposition de ses clients. Certaines de ces maisons sont anciennes pour un domaine comme celui de la traduction et ont largement dépassé le quart de siècle (la multinationale de la traduction mise à part).

Pour vague que soit cette description du secteur des bureaux de traduction, elle semblera un modèle de précision en comparaison de celle qu'on pourrait tenter de faire du monde innombrable et varié des pigistes.

Pour les besoins de la cause, nous entendons par pigiste une personne ayant un emploi permanent, de traducteur ou d'une autre profession, et pour laquelle la traduction à la pige constitue une activité complémentaire, souvent simplement occasionnelle.

Le dénombrement des pigistes est impossible pour la simple raison que ceux-ci échappent à tout contrôle. Tout ce qu'on peut dire, c'est qu'ils sont très nombreux. Sur les quelque 400 membres agréés inscrits au répertoire 1975 de la Société des traducteurs du Québec, environ 60 pour cent d'entre eux déclarent accepter des travaux à la pige. La proportion est peut-être moindre parmi les membres adhérents, au nombre d'environ 500 . On peut cependant penser qu'au moins 350 membres de la S.T.Q. font de la traduction à la pige, presque tous à Montréal et en grande majorité de l'anglais au français. Et la S.T.Q. est, malheureusement, bien loin de regrouper tous les traducteurs! 


\section{LES BUREAUX DE TRADUCTION}

Un trait semble caractériser avant tout le bureau de traduction, c'est la polyvalence. Les clients proviennent en cffet d'à peu près tous les domaines de l'activité économique, technique ou autre. Il va sans dire que cette variété de domaines n'est pas sans présenter de graves inconvénients. Il est en effet impossible de disposer, dans un même bureau, de traducteurs compétents dans des domaines aussi différents que la pharmacie et l'assurance, le droit et l'électronique, d'autant plus que, à moins d'avoir une clientèle attitrée dans un secteur particulier, les demandes sont imprévisibles. Sans parler de la documentation énorme qu'il faudrait avoir sous la main pour couvrir tous ces domaines. Dans de tels cas, le bureau de traduction qui veut faire du travail sérieux n'a d'autre ressource que de faire appel à des pigistes spécialisés.

Cette situation a fait qu'une certaine spécialisation - par exemple, finances et comptabilité, certains domaines de la technique, médecine et pharmacie, etc. se manifeste depuis quelques années parmi les bureaux de traduction petits et moyens. Elle est à l'avantage de tous : du bureau, auquel elle facilite le travail et le rend plus rentable, et de l'acheteur, auquel elle assure meilleur service et meilleure qualité. Cette spécialisation s'est produite, d'une part, du fait que certains bureaux ont eux-mêmes voulu se limiter à des domaines qu'ils connaissent et, d'autre part, du fait que les acheteurs se sont éduqués, notamment sous l'impulsion de leurs services de traduction. Ils comprennent mieux les problèmes de la traduction et deviennent plus difficiles sur la qualité. Si une évolution devait se produire dans notre domaine, il serait à souhaiter qu'elle aille dans le sens de la spécialisation.

Aux difficultés entraînées par la variété des sujets, vient s'en ajouter une autre : celle de la rentabilité. Le travail de traduction sérieux demande du temps, tous les traducteurs le savent. Recherche de la documentation, particulièrement longue pour les sujets nouveaux, traduction proprement dite, révision, dactylographie, relecture, correction d'épreuves, éventuellement, sont les différentes phases chronophages - si on nous permet ce néologisme - qui mènent au produit fini.

Là encore, les chiffres manquent, mais les salaires - ceux des traducteurs et du personnel auxiliaire - constituent de toute évidence la majeure partie du prix de revient de la traduction, dans lequel entre aussi naturellement les frais généraux habituels.

Dans ces conditions, la rentabilité de l'entreprise repose presque essentiellement sur le rendement des traducteurs. Rapidité, universalité et qualité dans le travail doivent donc caractériser les traducteurs d'un tel bureau, plus que de tout autre secteur. Il va donc sans dire que le recrutement du personnel des bureaux de trađuction pose certains problèmes. D'autant plus que, si le travail dans un bureau de traduction est intéressant, principalement du fait de sa variété, il est également épuisant par suite de la cadence de travail imposée, d'abord par la nécessité du rendement, ensuite par les délais d'exécution sans rapport avec la réalité souvent imposés par les clients. La mobilité des traducteurs cause d'autres difficultés de personnel. D'une part, la demande de bons traducteurs est grande et ceux-ci ont souvent des occasions d'avancement dans des entreprises ou des bureaux concurrents. D'autre part, nombre d'entre eux sont tentés de se mettre à leur compte. Il 
arrive donc fréquemment que des bureaux de traduction voient partir des membres de leur personnel qu'ils ont mis des années à former. Un placement s'évanouit du jour au lendemain.

La recherche d'une certaine assurance de rentabilité a amené quelques bureaux de traduction à tenter la facturation à l'heure plutôt que la traditionnelle facturation au mot. La méthode est certes avantageuse pour le fournisseur, et elle peut l'être pour le client si la traduction est exécutée rapidement. Il semble bien toutefois qu'on ne puisse l'appliquer qu'à des clients bien au fait des problèmes de la traduction et ayant des relations établies de longue date avec leur fournisseur. Dans le cas de la facturation à l'heure, le devis est évidemment plus difficile à établir, le prix au mot ne pouvant éventuellement servir que de point de repère.

Étant donné les différents facteurs qui viennent d'être évoqués, spécialisation comprise, il est souvent rentable pour le bureau de traduction de faire appel à des pigistes. Dans ce cas, le prix de revient du travail de traduction lui-même est connu de façon précise. Le temps d'exécution du travail n'intervient plus. Si l'on a su choisir un traducteur de qualité, la révision sera réduite au minimum. De plus, on pourra confier les travaux à des spécialistes de domaines que le bureau de traduction n'aborde pas habituellement.

Rien d'étonnant alors que les bureaux de traduction fassent si largement appel aux pigistes. Certains font même exclusivement faire leurs travaux par ces traducteurs et leur rôle se résume souvent, on le sait, à celui d'intermédiaires. Dans le cas de longs travaux pour lesquels les délais d'exécution sont suffisants, quelques bureaux s'adressent même à des maisons ou pigistes établis à l'étranger, incités surtout en cela par les différences de tarifs existant entre le Canada et New York, Paris ou Genève. Toutefois, aussi soignées que les traductions faites dans ces villes puissent être, elles laissent souvent à désirer sur bien des points car les traducteurs ne sont pas au courant des innombrables particularités du milieu auquel elles sont destinées. Dans ce cas, une révision minutieuse s'impose et elle fait perdre ce qui a été gagné sur le prix de la traduction. Que les traducteurs canadiens se rassurent, en grande partie, la traduction n'est pas une marchandise importable.

\section{LES PIGISTES}

Les pigistes forment un groupe apparemment très nombreux mais aussi très varié quant aux domaines de la traduction et des langues de travail. La grande majorité des traductions en langues autres que le français et l'anglais sont, au Canada, l'ouvre de pigistes, mises à part celles sortant du service des langues étrangères du Secrétariat d'État.

Les pigistes peuvent se diviser en trois catégories: les professionnels, les semi-professionnels et les amateurs.

Les professionnels sont ceux pour qui la traduction est un métier; les semiprofessionnels sont ceux pour qui la traduction constitue une activité secondaire mais qui l'exercent dans un domaine particulier où ils ont des connaissances spécialisées, par exemple, un professeur de langues, un ingénieur ou un médecin. 
Les amateurs sont les autres. Cette dernière catégorie comprend beaucoup de personnes que la traduction intéresse et auxquelles la pige donne un moyen de débuter dans le métier. Nombre d'entre elles se retrouvent parmi les membres des sociétés de traduction ou les élèves des cours du soir et elles manifestent ainsi leur désir de se perfectionner. On ne compte plus les bons traducteurs qui ont suivi cette filière pour accéder à la profession.

Malgré tout, la catégorie comprend encore trop de sujets sans connaissances suffisantes, souvent même peu doués pour le travail qu'ils entreprennent et ne manifestant d'autre part aucun désir de perfectionnement. Ces personnes sont employées - ou exploitées - par des acheteurs de traduction au rabais pour qui seul compte le fait d'offrir la notice, l'emballage ou l'enseigne en français que la loi les oblige désormais à présenter, et pour qui la qualité est le moindre des soucis. Ces acheteurs sont en fait bien plus à blâmer que les « traducteurs » qu'ils emploient. La traduction semble si facile - ne suffit-il pas de savoir un peu d'anglais et d'écrire un peu en français pour traduire, n'est-ce pas ? - et on peut en faire si aisément à domicile, qu'il n'est pas étonnant de voir tant d'amateurs la pratiquer.

Le secteur des traducteurs pigistes, professionnels et semi-professionnels tient, on l'a vu, une place importante dans le monde de la traduction. Au cours des dernières années, s'est constitué à Montréal (pour ne citer que cette ville) un groupe de traducteurs pigistes tel qu'on n'en trouve certainement peu dans les autres grandes villes francophones.

Ces pigistes sont connus, ils demandent généralement une rémunération élevée mais en rapport avec leur compétence, et beaucoup d'entre eux ont leurs clients attitrés. Généralement spécialisés, c'est à eux, aussi, qu'on fait appel en cas d'urgence... et bien souvent, le traducteur pigiste n'a pas de questions à se poser sur l'emploi du temps de ses soirées ou de ses fins de semaine !

Souvent, ces traducteurs disposent d'une documentation abondante dans leur domaine... et ils ont l'avantage d'avoir accès à celle de leur employeur, généralement bien fournie.

Ainsi, le pigiste, artisan du monde de la traduction, joue un rôle dont l'ampleur est difficile à évaluer, mais qui est certes essentiel.

\section{EVOLUTION ET AVENIR}

Le bureau de traduction a subi peu de changements au cours des 20 ou même 25 dernières années. Opération intellectuelle, la traduction se pratique à peu près toujours de la même façon depuis de longues années. Seule la généralisation de la dictée, rendue possible par l'enregistrement magnétique, a quelque peu modifié les habitudes de travail des traducteurs. Ceux-ci ont également désormais à leur disposition, du moins dans les grandes entreprises et les grands bureaux de traduction, des documentalistes qui peuvent les aider dans leurs recherches terminologiques. $\grave{A}$ moins de l'avènement de la traduction automatique, qui ne surviendra probablement pas dans un avenir rapproché, il est probable que peu de changements se produiront dans le domaine. 
On peut s'attendre toutefois, et c'est ce que souhaitent tous les traducteurs, à l'emploi de moyens d'exploitation plus rationnels et plus pratiques de la matière abondante amassée par les centres de documentation. Les banques de terminologie, interrogées directement par terminal, pourraient satisfaire en grande partie à ce besoin. Elles seraient d'un immense secours pour les bureaux de traduction et contribueraient sans aucun doute à l'amélioration de la qualité des travaux comme de la rentabilité.

Quant à l'organisation de la profession elle-même, sans vouloir jouer les prophètes, elle ne semble pas appelée à une grande évolution dans les prochaines années. Seule la reconnaissance professionnelle, en particulier celle donnant l'exclusivité de l'acte, pourrait apporter d'importants changements, voire même des bouleversements dans le métier. Mais on connaît les difficultés rencontrées sur le chemin de cette reconnaissance.

Quant au marché, les traducteurs savent aussi par expérience qu'il est très actif présentement. Les efforts de francisation, entraînés en particulier par la politique de bilinguisme du gouvernement fédéral et par la promulgation de la Loi 22 , ont apporté beaucoup d'eau au moulin des bureaux de traduction et des pigistes. Étant donné le nombre de documents existants déjà qui devront être traduits, et le fait que l'on continue encore à rédiger de multiples documents en anglais, la demande de traduction de l'anglais vers le français parait devoir demeurer forte pendant encore quelques années. Quant à la traduction du français vers l'anglais, elle est déjà beaucoup plus pratiquée qu'elle ne l'était il y a seulement deux ou trois ans et il semble bien que la tendance doive s'intensifier du fait qu'on rédigera de plus en plus en français.

Cette généralisation de la rédaction en français ne doit d'ailleurs pas inquiéter les traducteurs de l'anglais vers le français. En effet, du fait de la pénurie de bons rédacteurs techniques ou autres, il arrive de plus en plus fréquemment qu'on fasse appel à des traducteurs, sinon pour rédiger, du moins pour réviser des textes français. Cela n'a rien d'étonnant car, de nos jours, qui d'autre que le traducteur apprend à rédiger correctement ?

Aucune évolution marquante ne semble donc en vue en ce qui concerne les bureaux de traduction. Étant donné leur nombre relativement élevé et le grand nombre de pigistes, il est peu probable qu'on assiste à la formation de bureaux géants employant quelques dizaines de traducteurs. Ces bureaux seraient-ils viables ? L'usine à traduction est-elle souhaitable?

En revanche, il y a sûrement augmentation du nombre de bureaux de traduction, des traducteurs se mettant à leur compte et des non-traducteurs se mettant aussi de la partie. Par ailleurs, des entreprises pour lesquelles la traduction n'était qu'une activité tout à fait accessoire décident, elles aussi, de tirer parti de la manne qu'elles voient dans la Loi 22. C'est le cas de certaines agences de publicité qui ont créé des divisions, ou même de véritables filiales de traduction. Ces bureaux proposent leurs services aux clients de l'agence qui désirent franciser leur entreprise et qui doivent, par conséquent, faire traduire un grand nombre de documents de toutes sortes mais n'ayant toutefois rien de publicitaire. On assiste également à un phéno- 
mène curieux : les services de traduction de certaines grandes sociétés, qui constituent un centre de rentabilité à l'intérieur de l'entreprise, en viennent à proposer leurs services à des fournisseurs de ces sociétés et sont devenus en réalité de véritables bureaux de traduction. Cette multiplication des bureaux de traduction, traducteurs indépendants - et aussi des pigistes du fait de 1a formation d'un nombre toujours croissant de jeunes traducteurs - ne manquera pas d'avoir un effet sur le marché. La concurrence se fera plus âpre dans les années qui viennent, même si la demande est forte. Par ailleurs, l'éducation des acheteurs fera aussi que les exigences de qualité seront plus rigoureuses.

Notons en passant que, si les traducteurs sont groupés au sein d'associations très actives, il n'en va pas de même des bureaux de traduction qui, jusqu'à présent, n'ont pas encore jugé utile d'organiser leur secteur.

Il a déjà été proposé que des traducteurs indépendants ou des petits bureaux de traduction se regroupent en associations semblables à celles constituées par les avocats ou les comptables agréés. Cette formule présenterait de nombreux avantages, entre autres, une répartition possible des travaux selon les spécialités des divers associés, une distribution des charges de travail, en particulier au moment des vacances ou des absences, le partage de certains frais généraux, et aussi l'exploitation plus rationnelle de la documentation et des fichiers. Assisterons-nous à la création de ces cabinets de traduction ? Sans doute faudrait-il dans ce cas que les traducteurs indépendants sacrifient un peu de leur chère... indépendance. Mais, qui sait ? la conjoncture obligera peut-être à de tels regroupements qui seraient à la fois plus rationnels et mieux dans l'esprit de la profession que des bureaux de traduction géants.

ROGER BOIVINEAU 